\title{
Preservación postcosecha de Carica papaya L. por recubrimiento con bioplástico desarrollado a partir de almidón, arcilla y ajo
}

\author{
Postharvest preservation of Carica papaya $L$. by coating with \\ bioplastic developed from starch, clay and garlic.
}

\author{
Melissa Batista ${ }^{1 *}$, Maldonado Natasha ${ }^{1}$, Yailin Moreno ${ }^{1}$, Leticia Solís ${ }^{1}$, Rosa Quintero ${ }^{2}$, Jhonny Correa ${ }^{2}$, \\ ${ }^{1}$ Licenciatura en Ingeniería en Alimentos ${ }^{2}$, Grupo de Ciencia y Tecnología Innovadora de Alimentos (CYTIA) - Facultad de \\ Ciencia y Tecnología - Universidad Tecnológica de Panamá
}

\begin{abstract}
Resumen La vida útil postcosecha de algunas frutas climatéricas como la papaya es relativamente corta y en los periodos de abundancia comercial de dicha fruta las cantidades que se pierden son de magnitudes lamentables. Esto ha motivado diversas investigaciones enfocadas en el desarrollo de estrategias que permitan extender el periodo de consumo de esta fruta. En este estudio se desarrolló bioplásticos a partir de almidón de papa modificados con arcilla y ajo. La capacidad de los bioplásticos elaborados para retardar la maduración natural de la papaya fue evaluada, resultando que las papayas recubiertas con los bioplásticos prototipos se maduraron más lentamente que las papayas sin recubrimiento, y la incorporación de ajo en el bioplástico mantuvo en mejor estado las papayas durante 16 días.
\end{abstract}

Palabras clave Almidón de papa, glicerina, bioplástico, arcilla, ajo, temperatura.

\begin{abstract}
The postharvest lifespan of some climacteric fruits such as papaya is relatively short and in the periods of commercial abundance of this fruit the quantities that are lost are of regrettable magnitudes. This has motivated diverse investigations focused on the development of strategies that allow extending the period of consumption of this fruit. In this study, bioplastics were developed from potato starch modified with clay and garlic. The capacity of the bioplastics elaborated to retard the natural maturation of the papaya was evaluated, resulting that the papayas covered with the bioplastics prototypes matured more slowly than the uncoated papayas, and the incorporation of garlic in the bioplastic maintained the papayas in a better state for 16 days.
\end{abstract}

Keywords Potato starch, glycerin, bioplastic, clay, garlic, temperature.

* Corresponding authors: melissamakely26@gmail.com

\section{Introducción}

Estudios internacionales han estimado que del 30 al 50\% de los alimentos producidos en el planeta se pierden sin ser consumidos. Las razones por las pérdidas varían según el nivel de desarrollo tecnológico de los países, los tipos de alimentos y otros factores [1]. En Panamá se ha estimado que la pérdida de frutas y hortalizas en el mercado de abastos es de 30 toneladas diarias, mientras que la pérdida mensual ronda las 900 toneladas [2]. Entre las frutas susceptibles a las pérdidas se encuentra la papaya, la cual debido a su rápida maduración natural conduce a un corto periodo para la comercialización en el que además es susceptible a varios agentes parasíticos entre las que destaca el daño ocasionado por el hongo Colletotrichum gloeosporioide responsable de la enfermedad de la papaya conocida como antracnosis [3, 4].
El aumento de la vida útil y mantener de la calidad de frutas frescas ha sido objeto de estudios por muchos años. La aplicación de recubrimientos comestibles a frutas ha mostrado reducir el paso del oxígeno requerido para la respiración y así el proceso de maduración ocurre más lentamente. Adicionalmente, la capa del recubrimiento disminuye la pérdida de agua al actuar como una barrera moderada en la que se pueden incorporar agentes antimicrobianos o antioxidantes [5].

La incorporación de arcilla a películas biodegradables basadas en quitosano ha demostrado disminuir la permeabilidad de gases como el agua y el oxígeno [6]. La utilización de arcilla en elaboración de película a base de almidón de yuca influye en el grado de permeabilidad de los gases [7]. 
Mientras que el ajo ha sido ampliamente investigado por su actividad bactericida con buenos resultados [8,9], su actividad fungicida ha sido pobre. Esto quizás se debe a que las investigaciones conducidas se han enfocado mayormente en organismos patógenos hacia los humanos, de los que solo ciertas especies han mostrado susceptibilidad.

En este estudio se evaluó el potencial de bioplásticos elaborados a partir de almidón de papa, arcilla y ajo sobre la preservación de papayas al ser aplicados en forma de recubrimiento sobre las mismas por 16 días.

\section{Materiales y métodos}

\subsection{Materia prima}

Se obtuvo el ajo y los frutos de papaya completamente desarrollados del Supermercado Rey, ubicado en el Centro Comercial El Dorado, donde se habían mantenido almacenados por dos días. La arcilla modificada, montmorillonita conocida comercialmente como (Cloisite ${ }^{\circledR N a}+$ ) fue obtenida de Southern Clay Products Inc. (Texas, USA), el almidón de papa fue suministrado por cortesía del Grupo de Ciencia y Tecnología de los Alimentos de la Universidad Tecnológica de Panamá.

\subsection{Elaboración de bioplásticos y recubrimientos de frutas}

Las formulaciones de los bioplásticos, objeto de estudio, se indican en la tabla 1. Para su preparación, el agua fue calentada hasta obtener una temperatura entre 70 y $90^{\circ} \mathrm{C}$, luego se agregó el almidón poco a poco hasta su total solubilización y posteriormente se adicionaron los demás componentes. Las mezclas se dejaron enfriar hasta que alcanzaran la temperatura ambiente y se procedió a sumergir las papayas (previamente pesadas en una balanza de precisión electrónica PCE-WS 30) en cada mezcla respectivamente. Las papayas recubiertas se dejaron secar por 48 horas y los resultados fueron observados durante 16 días.

Tabla 1. Composición de los bioplásticos elaborados

\begin{tabular}{|c|c|c|c|c|c|}
\hline Bioplástico & $\begin{array}{c}\text { Almidón } \\
(\mathbf{g})\end{array}$ & $\begin{array}{c}\text { Glicerina } \\
(\mathbf{m L})\end{array}$ & $\begin{array}{c}\text { Agua } \\
(\mathbf{m L})\end{array}$ & $\begin{array}{c}\text { Arcilla } \\
(\mathbf{g})\end{array}$ & $\begin{array}{c}\text { Ajo } \\
(\mathbf{g})\end{array}$ \\
\hline Control & 0 & 0 & 100 & 0 & 0 \\
\hline Simple & 5 & 5 & 95 & 0 & 0 \\
\hline Arcilla & 5 & 5 & 95 & 0.2632 & 0 \\
\hline Arcilla y Ajo & 5 & 5 & 95 & 0.2632 & 0.2632 \\
\hline
\end{tabular}

\subsection{Determinación de cambios físicos en las papayas recubiertas con bioplásticos}

Las papayas recubiertas se mantuvieron a una temperatura de $20.7^{\circ} \mathrm{C} \pm 0.3{ }^{\circ} \mathrm{C}$ y una humedad de $67.0 \% \pm 4.7 \%$. Para la determinación de la pérdida de peso, cada una de las frutas recubiertas fue pesada en una balanza de precisión electrónica PCE-WS 30. Las mismas fueron pesadas al inicio y al final del periodo de almacenamiento. Durante el periodo de almacenamiento se dio seguimiento a variaciones típicas de la cáscara indicativas de la calidad de los frutos como: aparición de coloración amarilla-naranja, hundimiento en el área de la cáscara, aparición de manchas chocolates y crecimiento microbiano. Estas observaciones se documentaron mediante fotografías.

\section{Resultados y discusión}

El recubrimiento de las papayas con bioplásticos demostró disminuir su maduración, lo que se ilustra en la figura 1. Resultados similares se han logrado con diferentes bioplásticos [5], [10]. Al tercer día las papayas sin recubrimiento (control) mostraron diferencias con respecto a las recubiertas; las primeras habían desarrollado una coloración amarilla, indicativa de un avance normal en el grado de maduración, mientras que las papayas recubiertas se mantenían más verdes.

El proceso de maduración de las papayas sin recubrimiento alcanzó su estado máximo para consumo a los nueves días, a partir de los cuales se apreció un marcado crecimiento microbiano, haciendo estas papayas no recomendables para consumo a partir del día diez de almacenamiento. En contraste, en las papayas recubiertas con bioplásticos no se apreciaba desarrollo de coloración amarilla ni crecimiento microbiano.

No obstante, las cáscaras de las papayas recubiertas con bioplástico simple y con arcilla cambiaron de tener una apariencia lisa y uniforme a una rugosa y con hundimientos en algunas áreas; esta observación se hizo más notoria a partir del día 12. El comportamiento descrito en la observación anterior no fue observado en las papayas recubiertas con la formulación del bioplástico que incluye ajo. Esto sugiere que la rugosidad y el hundimiento en la superficie de las papayas están asociados a la degradación causada por microorganismos, la cual es reducida por la actividad antimicrobiana del ajo [8,9].

En el día 16 las frutas fueron cortadas e inspeccionadas en la parte interior (figura 2). Las papayas recubiertas con bioplástico simple (figura 2a) y con arcilla (figura 2b) presentaban acumulación de agua y mal olor, evidencias características de descomposición de la materia orgánica. A diferencia de las papayas recubiertas con bioplástico que incluye ajo se conservaron sin degradación en su interior (figura 2c), lo que concuerda con lo observado en el exterior de las frutas correspondientes a los 16 días (figura 1). 
Solís (et al): Preservación postcosecha de Carica papaya L. por recubrimiento con bioplástico desarrollado a partir de almidón, arcilla y ajo

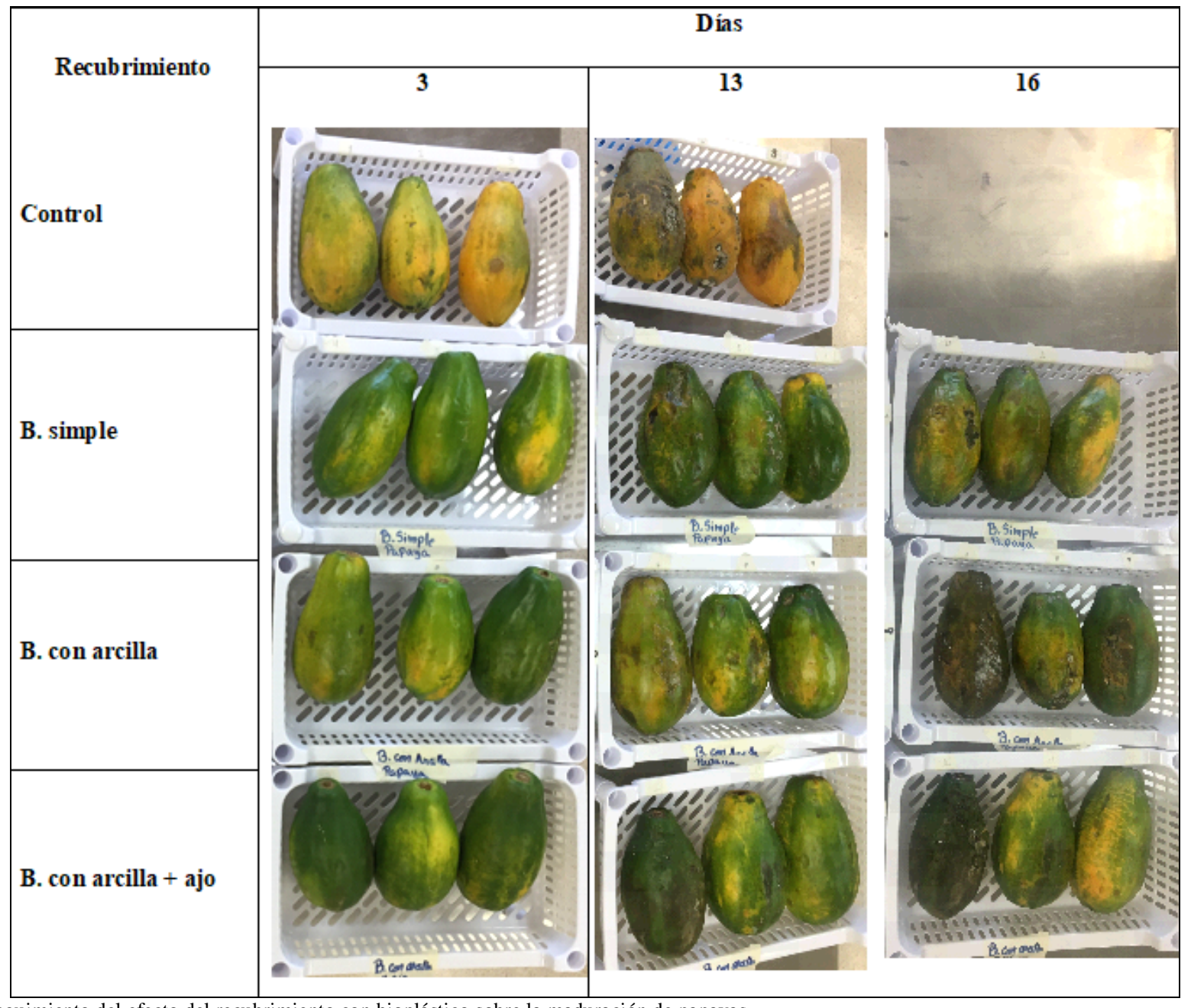

Figura 1. Seguimiento del efecto del recubrimiento con bioplástico sobre la maduración de papayas.

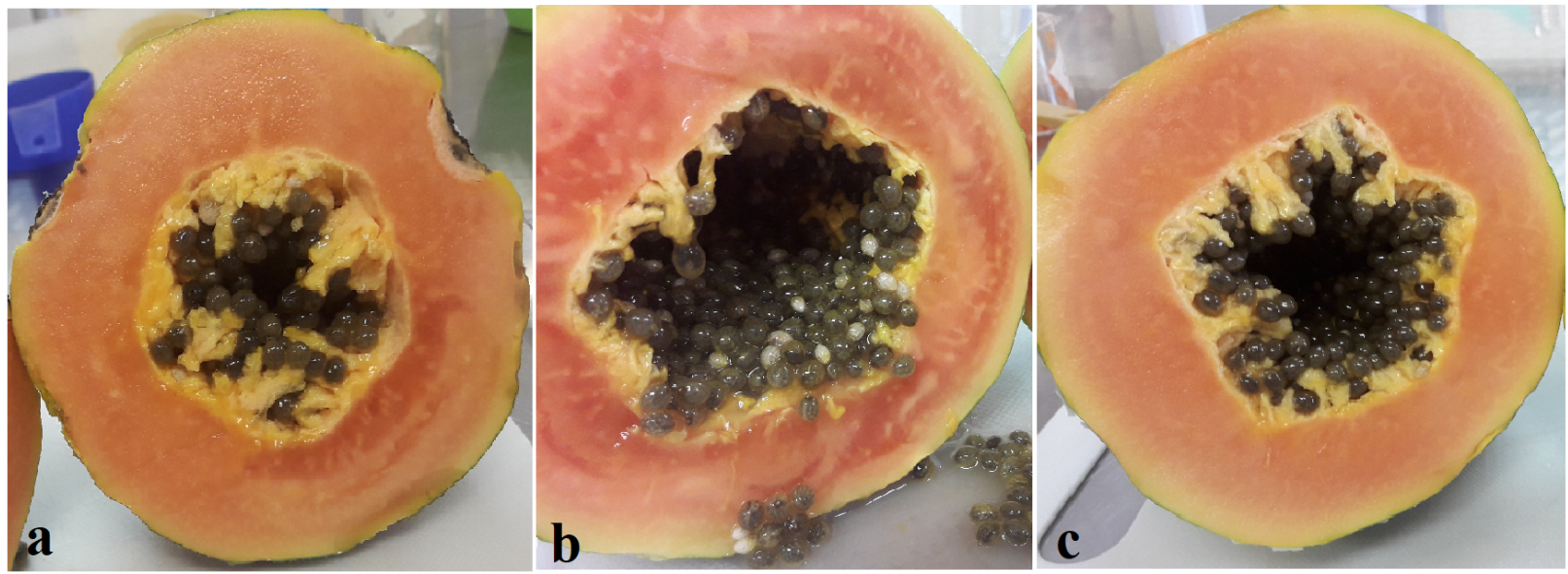

Figura 2. Apariencia del interior de ejemplares de las papayas recubiertas con bioplástico simple (a), con arcilla (b) y con arcilla y ajo (c). 
La reducción de peso observada en las papayas recubiertas con bioplástico muestra que el bioplástico elaborado evitó la transferencia de masa desde el interior de la fruta hacia el exterior (figura 3). Esto sugiere que el retardo en la maduración se debe a la inhibición de la respiración debido a que el bioplástico actuó como barrera para penetración del oxígeno. Esto está de acuerdo con las observaciones reportadas en la literatura $[5,10]$ y es indicativo de que la descomposición de la materia orgánica en el interior de las papayas evidenciada en los ejemplares recubiertos por las formulaciones de bioplástico sin ajo, descrito arriba, se debe a fermentación anaeróbica.

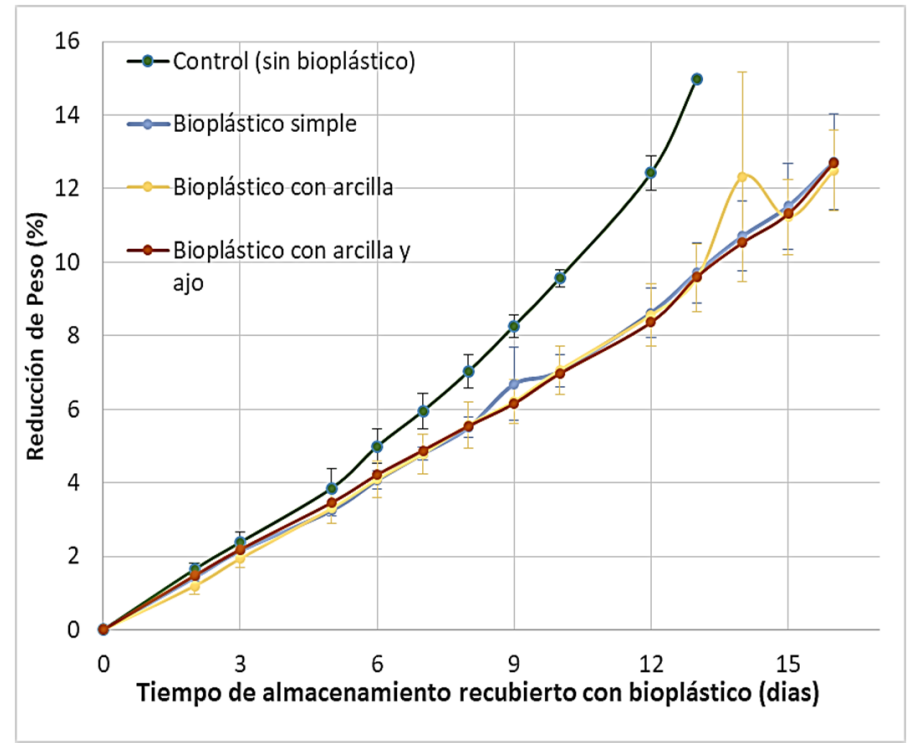

Figura 3. Pérdida de peso registrada para las papayas. Las barras de error muestran la desviación estándar del promedio de mediciones a tres frutos distintos.

Considerando que no hay diferencia apreciable entre la reducción de peso de las papayas recubiertas con bioplásticos simples o con arcilla (figura 3), concluimos que la incorporación de arcilla, en la concentración utilizada en la formulación de bioplástico, no proporcionan un beneficio en cuanto a la permeabilidad de gases. El uso de arcilla en bioplásticos no ha sido ampliamente estudiado para recubrimiento de frutas [7].

\section{Conclusión}

La utilización de bioplástico a base de almidón de papa como recubrimiento de papaya disminuye el tiempo para alcanzar la maduración y una reducción en la pérdida de peso. La incorporación de arcilla a la formulación de bioplástico no aportó beneficios a la preservación de papayas, por el contrario, favoreció la degradación en su interior. Esto no se observó cuando la formulación incluyó adicionalmente ajo, por lo que el ajo es un conveniente agente antimicrobiano para la preservación de papaya.

\section{AGRADECIMIENTO}

Los autores le agradecen al Lic. Alejandrino Sevillano y al departamento de química de la Universidad Tecnológica de Panamá, por su apoyo en la recolección de datos, en el desarrollo de proyectos de investigación e innovación.

\section{REFERENCIAS}

[1] Porat, R., Lichter, A., Terry, L., Harker, R., \& Buzby, J. (2018). Postharvest losses of fruit and vegetables during retail and in consumers' homes: Quantifications, causes, and means of prevention. Postharvest Biology and Technology(139), 135-149.

[2] Tejera, A. (2017, Ago 20). Frutas, vegetales y horatalizas terminan tirados en la basura. La Prensa. Retrieved septiembre 13, 2018, from https://impresa.prensa.com/panorama/Frutas vegetaleshortalizas-terminan-tirados_0_4829517056.htm.

[3] Paull, R., Nishijima, W., \& Reyes, M. a. (1997). Postharvest handling and losses during marketing of papaya (Carica papaya L.). Postharvest Biology and Technology(11), 165-179.

[4] Maqbool, M., Ali, A., Alderson, P. G., Muda Mahamed, Y. S., \& Zahid, N. (2011). Postharvest application of gum arabic and essential oils for controlling anthracnose and quality of banana and papaya during cold storage. Postharvest Biology and Technology(62), 71-76.

[5] Chander Mahajan, B. V., Tandon, R., \& Kapoor, S. a. (2018). Natural Coatings for shelf-life enhancement and quality maintenance of fresh fruits and vegetables. Journal of Postharvest Technology, 1(06), 12-26.

[6] Garde, G. (2014). Efecto de la adición, tipo y concentración de nanoarcilla sobre las propiedades mecanicas y de barrera de películas biodegradables basadas en quitosano. Uztaila: Universidad Pública de Navarra. Retrieved from http://academica-

e.unavarra.es/bitstream/handle/2454/12224/Gaizka\%20_Garde\% 20\%20TyC\%20-IAA.pdf?sequence $=1$

[7] Leal Oliveira, V. R., Noberto Xavier, T. D., Oliveira Araújo, N., Lima Almeida, J. G., Mendes Aroucha, E. M., Gomes Santos, F. K., \& de Lima Leite, R. H. (2017). Evaluation of Biopolimeric Films of Cassava Starch with Incorporation of Clay Modified By Ionic Exchange and its Application as a Coating in a Fruit. Materials Research, 2(20), 758-766.

[8] Pranoto, Y., \& Salokhe, V. a. (2005). Physical and antibacterial properties of alginate-based edible film incorporated with garlic oil. Food Research International(38), 267-272.

[9] Gaitán, I., Paz, A., Zacchino, S., Tamayo, G., Giménez, A., \& Pinzón, R. C. (2011). Subcutaneos antifungal screening of Latin American Plant extracts against Sporothrix schenckii and Fonsecaea pedrosoi. Pharmaceutical Biology, 9(49), 907-919.

\section{$12 \mid$ RIC}


Solís (et al): Preservación postcosecha de Carica papaya L. por recubrimiento con bioplástico desarrollado a partir de almidón, arcilla y ajo

[10] Ali, A., Muda Muhammad, T., \& Sijam, K. y. (2011). Effect of chitosan coating on the physicochemical characteristics of Eksotika II papaya (Carica papaya L.) fruit during cold storage. Food Chemistry(124), 620-626. Retrieved from Effect of chitosan coatings on the physicochemical characteristics of. 